\title{
LH-RH analogues in the treatment of young women with early breast cancer: Long-term follow-up of a phase II study
}

\author{
FRANCESCO RECCHIA $^{1,2}$, STEFANO NECOZIONE $^{3}$, MASSIMO BRATTA $^{2}$, \\ MICHELE ROSSELLI ${ }^{4}$, GABRIELE GUERRIERO ${ }^{2}$ and SILVIO REA ${ }^{2,5}$ \\ ${ }^{1}$ Oncology Unit, Civilian Hospital, Avezzano; ${ }^{2}$ Carlo Ferri Foundation, Monterotondo, Roma; \\ ${ }^{3}$ Clinical Epidemilogy, University of L'Aquila, L'Aquila; ${ }^{4}$ Oncology Unit Civilian Hospital, Frascati; \\ ${ }^{5}$ Surgical Oncology, University of L'Aquila, L'Aquila, Italy
}

Received September 7, 2014; Accepted October 27, 2014

DOI: 10.3892/ijo.2014.2811

\begin{abstract}
To prevent premature ovarian failure (POF), high-risk, premenopausal women with early breast cancer were given a luteinizing-hormone releasing hormone (LH-RH) analogue during adjuvant chemotherapy. After an adriamycin-based regimen, patients received radiation therapy concomitant with cyclophosphamide, methotrexate and 5-fluorouracil. An aromatase inhibitor was given to patients positive for the estrogen receptor $\left(\mathrm{ER}^{+}\right)$. The median age was 43 years (range, 26-45). Among 200 consecutive patients, 46\% had no axillary node, and $54 \%$ had a mean of 5.4 positive nodes (range, 1-25); $56 \%$ were $\mathrm{ER}^{+}, 44 \%$ were estrogen receptor negative (ER'), $13 \%$ were triple negative, and 20 had tumors positive for the oncogene, c-erb-B2 (identified with fluorescent in situ hybridization). After a median follow-up of 105 months (range, 65-180), no patient under 40 years old exhibited POF, while $44 \%$ of patients over 40 years old exhibited POF. Eight pregnancies were recorded: 7 at term and 1 voluntary interruption. The 10-year disease-free survival and overall survival rates were 85 and $91 \%$, respectively. These data showed that, in premenopausal patients with early breast cancer, the addition of an LH-RH analogue to adjuvant chemotherapy was well tolerated, prevented POF, and was associated with excellent disease-free survival and overall survival rates.
\end{abstract}

\section{Introduction}

Breast cancer in young women is associated with a poor prognosis; in the absence of adjuvant treatment, approximately half of the patients will die of metastatic disease. Ovarian ablation was the first described form of endocrine therapy for treating advanced breast cancer (1). Previous trials have also suggested

Correspondence to: Dr Francesco Recchia, Via Del Castagneto 15, 67056 Luco dei Marsi (AQ), Italy

E-mail: frecchia1946@libero.it

Key words: high-risk premenopausal early breast cancer, LH-RH analogues, adjuvant therapy, premature ovarian failure that ovarian ablation with surgery or radiation therapy could reduce the risk of recurrence in young premenopausal women (2). However, in recent years, adjuvant chemotherapy has become the standard treatment for young premenopausal patients with breast cancer that have a high risk of systemic disease (3).

One important aspect of chemotherapy is the toxicity, which can induce premature ovarian failure (POF). POF was reported in $68 \%$ of women after cyclophosphamide, methotrexate, and 5-fluorouracil (CMF) regimens, and a POF higher rate was observed in women treated with anthracycline-based regimens (4). This may be a serious problem for young women in reproductive ages. Preserving fertility during adjuvant chemotherapy has been an ongoing endeavor, since 1987 (data on file), when a nulliparous, just married, 33-year-old woman, with high risk of breast cancer underwent anthracycline-based adjuvant chemotherapy. She was treated with buserelin, a luteinizing-hormone releasing hormone (LH-RH) agonist, once daily in a nasal spray, during 6 months of chemotherapy. After chemotherapy and radiation therapy, she completed two full term pregnancies.

In the following years, we conducted a clinical trial in premenopausal patients with early breast cancer, where another LH-RH agonist, goserelin was given subcutaneously (3.6 mg) every 28 days for 1 year, in addition to adjuvant chemotherapy. In the early course of that study, we found that the treatment was well tolerated and it protected long-term ovarian function (5). In the second part of the study, additional patients were recruited to receive $11.25 \mathrm{mg}$ goserelin every 84 days for 2 years. After a median follow-up of 48 months, patients that had received goserelin for 2 years showed a lower rate of POF compared to controls, which was the main objective of the study. Those patients also had a statistically significant $(\mathrm{P}<0.005)$ improvement in diseasefree survival (DFS) compared to patients treated for 1 year (6). Furthermore, we found that the majority of local-regional and systemic recurrences occurred during the second year of the study in patients with estrogen-receptor-positive $\left(\mathrm{ER}^{+}\right)$ tumors. Therefore, we amended the protocol: the LH-RH analogue therapy was continued for two more years, and an aromatase inhibitor was added to the regimen for patients with $\mathrm{ER}^{+}$tumors. With this background, and according to the latest 
data from the literature (7), the last 100 patients that entered the study received anthracycline-taxane-based chemotherapy, concomitant with goserelin therapy, continued for 5 years. Here, we report the long-term clinical outcome of all 200 patients treated with ovarian protection during chemotherapy, focusing on the outcome of patients treated with goserelin for 5 years or less.

\section{Materials and methods}

Patients. Eligibility in the trial required documented premenopausal status with follicle stimulating hormone (FSH) levels $<10 \mathrm{mU} / \mathrm{ml}, 17-\beta$ estradiol levels between 20 and $693 \mathrm{pg} / \mathrm{ml}$, and progesterone levels between 0.15 and $28 \mathrm{ng} / \mathrm{ml}$. Inclusion criteria comprised histologically proven breast cancer confined to the breast and ipsilateral axilla (tumor-node-metastasis classifications: T2-T3, N0-N3 and M0), unilateral or bilateral, with positive or negative expression of the estrogen and progesterone receptors $\left(\mathrm{ER}^{+}\right.$and $\mathrm{PGR}^{+}$or $\mathrm{ER}^{-}$and $\mathrm{PGR}^{-}$, respectively). Receptor expression was determined by immunohistochemical testing of tumor biopsies; results were considered positive when expression was detected in $>10 \%$ of tumor cells. All included women had undergone a modified radical mastectomy or breast-conserving surgery plus full axillary node dissection, followed by radiation therapy. All patients had Eastern Co-operative Oncology Group scores of 0 to 1 for performance status. Macroscopic metastatic spread of the disease was excluded according to the usual criteria. The following laboratory parameters were required: granulocyte count $\geq 2,000 / \mu 1$, platelet count $\geq 100,000 / \mu 1$, hematocrit $\geq 30 \%$, total bilirubin and alanine aminotransferase (AST) levels $\leq 1.5$ times the upper limit of normal, serum creatinine concentration $\leq 1.8 \mathrm{mg} / \mathrm{dl}$, and left ventricular ejection fraction $\geq 50 \%$. Patients were excluded when they had histologicallydocumented metastases or malignancies other than curatively treated skin or cervical cancer. All patients provided written informed consent. Patients underwent clinical follow-up examinations every 6 months. The study was performed according to the Declaration of Helsinki, after obtaining local Ethics Committee approval.

Treatment plan. The treatment of the first 100 patients was previously described $(5,6)$. At the start of the study, 3 weeks after surgery, and 1 week before starting chemotherapy, the second group of 100 patients received $11.4 \mathrm{mg}$ goserelin every 84 days for 5 years. Chemotherapy consisted of 4 courses of anthracycline-taxane therapy (epirubicin, $75 \mathrm{mg} /$ $\mathrm{m}^{2}$ and docetaxel, $75 \mathrm{mg} / \mathrm{m}^{2}$ ), followed by 6 courses of CMF (600 mg/m² cyclophosphamide, $600 \mathrm{mg} / \mathrm{m}^{2}$ 5-fluorouracil, and $40 \mathrm{mg} / \mathrm{m}^{2}$ methotrexate). Both regimens were repeated every 3 weeks, while the latter regimen was administered concomitant with radiation therapy.

Eleven patients with $>10$ positive axillary nodes and a median age of 37 years, were treated with high-dose chemotherapy and autologous, peripheral blood progenitor cell (PBPC) transplantations (6). For this protocol, 4 weeks after the fourth course of anthracycline-based chemotherapy, one course of high-dose chemotherapy (carboplatin, etopside and melphalan) was delivered together with previously collected progenitor cells. One to 2 months after PBPC transplantation, patients received 6 courses of CMF chemotherapy, administered every 21 days concomitant with radiation therapy.

Twenty patients with tumors positive for the oncogene, c-erb-B2, received trastuzumab. Following completion of chemotherapy, all patients with $\mathrm{ER}^{+}$tumors received the aromatase inhibitor, exemestane (10 $\mathrm{mg}$ daily), for 5 years. After aromatase inhibitor treatment, 28 high-risk patients with $\mathrm{ER}^{+}$tumors received $20 \mathrm{mg}$ tamoxifen for 5 more years (7).

During the study, all patients received supplements of vitamin $\mathrm{D}$, calcium and bisphosphonates. Radiation therapy was delivered to all patients $(74 \%$ treated with segmental mastectomies and $26 \%$ treated with modified radical mastectomy) concomitant with $\mathrm{CMF}$, after the anthracycline-based chemotherapy.

Statistical analysis. The date of relapse was defined as the day that recurrent disease was diagnosed. DFS was defined as the time from the first LH-RH dose to the diagnosis of any relapse, the appearance of a second primary cancer, or death, whichever occurred first. DFS and overall survival (OS) were estimated with the Kaplan-Meier, product-limit method (8). Survival curves of sub-groups of patients were compared with the log-rank test. Adverse events were monitored based on standard World Health Organization criteria (9).

Data analyses were performed in March 2014. All patients were evaluated according to the intention-to-treat principle.

\section{Results}

Patient characteristics. The present study included 200 patients that had been diagnosed with adenocarcinoma of the breast, stage PT2-3a, N-/+, M0 and had undergone a modified radical mastectomy or breast conserving surgery plus a full axillary node dissection. The first cohort of patients entered the study between September 1993 and August 2002; the second cohort entered between September 2002 and June 2007. The baseline demographics and tumor characteristics of the first and second cohorts are shown in Table I. All patients were fully evaluated for POF. All patients had a good performance status, and the median age was 43 years. All women were premenopausal at the time of breast cancer diagnosis; they had normal levels of gonadotropins, estradiol and progesterone. The first cohort comprised $42 \%$ of patients with N0; in the second part of the trial, the proportion with negative axillary nodes increased to $50 \%$. This increase was probably due to the lack of age-specific screening procedures and to recent refinements in diagnostic procedures. However, although this was not a randomized study, no statistically significant difference was observed between the two cohorts of patients in hormone receptor status $(\mathrm{P}<0.001)$, in tumor histology $(\mathrm{P}<0.001)$ or in clinical stage $(\mathrm{P}<0.001)$ at diagnosis. The first and second cohorts included 11 and 10 patients, respectively, with more than ten positive axillary nodes. Treatment compliance was excellent; all patients received the assigned treatments of the LH-RH analogue, chemotherapy, radiotherapy and hormonal therapy.

Fertility, time to progression and survival. After a median follow-up of 105 months (range, 65-180), all patients had completed chemotherapy and LH-RH analogue treatment. 
Table I. Patient and tumor characteristics.

\begin{tabular}{|c|c|c|}
\hline Characteristics & $\mathrm{N}$ & $\mathrm{N}$ \\
\hline Patients groups & $1-100$ & $101-200$ \\
\hline \multicolumn{3}{|l|}{ Age (years) } \\
\hline Median & 43 & 43 \\
\hline Range & $27-50$ & $26-45$ \\
\hline \multicolumn{3}{|c|}{ Hormone receptors status } \\
\hline $\mathrm{ER}^{+}$ & 52 & 56 \\
\hline $\mathrm{ER}^{-}$ & 48 & 44 \\
\hline \multicolumn{3}{|l|}{ Tumor histology } \\
\hline Ductal infiltrating & 76 & 85 \\
\hline Lobular infiltrating & 13 & 10 \\
\hline Other & 11 & 5 \\
\hline \multicolumn{3}{|l|}{ Grading } \\
\hline $\mathrm{G} 1-\mathrm{G} 2$ & 44 & 51 \\
\hline G3 & 56 & 49 \\
\hline \multicolumn{3}{|l|}{ Clinical stage } \\
\hline II A & 48 & 51 \\
\hline II B & 16 & 13 \\
\hline III A & 11 & 8 \\
\hline III B & 16 & 18 \\
\hline III C & 9 & 10 \\
\hline \multicolumn{3}{|l|}{ Nodes } \\
\hline 0 & 42 & 50 \\
\hline $1-3$ & 40 & 32 \\
\hline $4-9$ & 7 & 8 \\
\hline$>10$ & 11 & 10 \\
\hline \multicolumn{3}{|c|}{ Type of primary surgery } \\
\hline Mastectomy & 32 & 29 \\
\hline Quadrantectomy & 68 & 71 \\
\hline
\end{tabular}

When treatment was discontinued, a total of 132 women $(66 \%)$ resumed normal menses, with appropriate levels of FSH, LH, $17-\beta$ estradiol and progesterone. Moreover, all women under 40 years old resumed normal menses, including 10 women treated with high-dose chemotherapy and PBPC transplantation. One of the patients treated with high-dose chemotherapy and PBPC transplantation became pregnant and elected to terminate the pregnancy. Five years after chemotherapy and radiotherapy, 6 patients had 7 babies.

Six patients experienced local disease recurrence after a median of 40 months (range, 26-111). Recurrences were treated with additional loco-regional and systemic therapies, as necessary. All patients are currently disease-free, after a median of 90 months. Three women experienced contra-lateral breast cancer, with $\mathrm{ER}^{-}$and $\mathrm{PR}^{-}$tumors. They were salvaged with radical mastectomy, and all three patients have remained disease-free to date. Another 25 patients (12.5\%) experienced systemic recurrences: 10 died within the first 5 years, and the other 15 patients lived for a median of 98 months (range, 74-170 months). At 5 years, $89.5 \%$ of patients were free from breast cancer recurrence at a distant site. For patients with

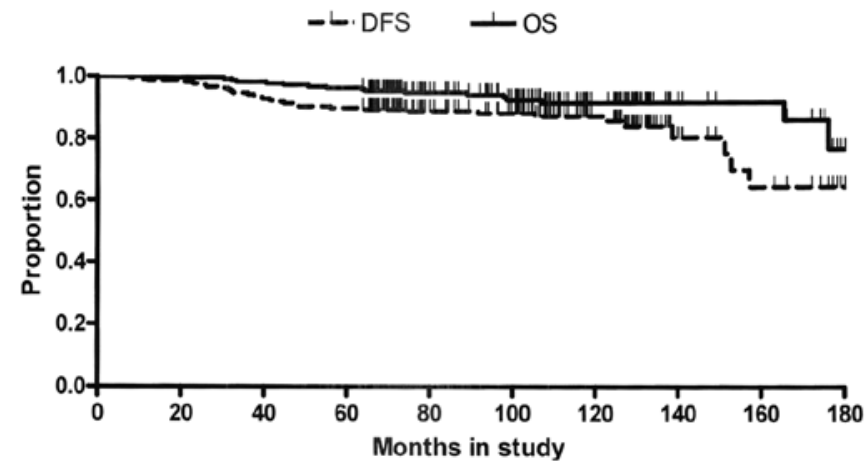

Figure 1. Survival rates. Disease-free survival (DFS - -): events 31 (15.5\%); censored 169 (84.5\%); 15-year DFS rate, 63.9\%. Overall survival (OS -): Events $17(8.5 \%)$; censored $183(91.5 \%)$; 15-year OS rate, $73.1 \%$. Median follow-up time, 105 months.

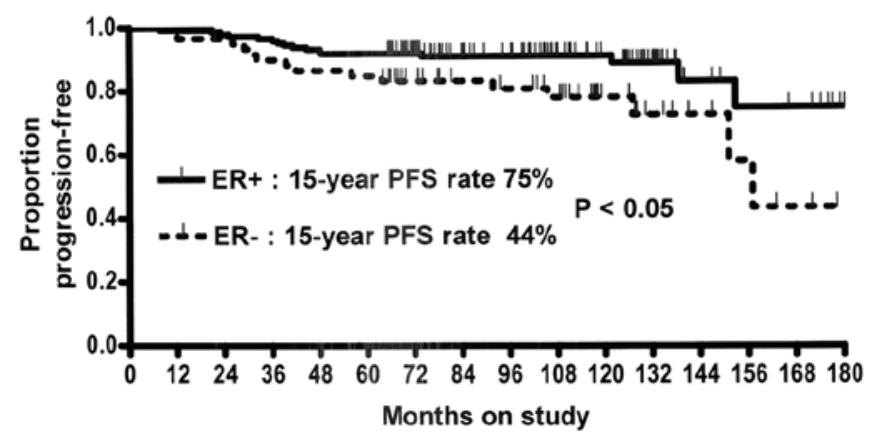

Figure 2. Disease-free survival (DFS - ) of patients with estrogen receptor positive tumors $\left(\mathrm{ER}^{+}\right)$. DFS (- -) of patients with estrogen receptor negative (ER') tumors; $\mathrm{P}<0.05$ (Log-rank test).

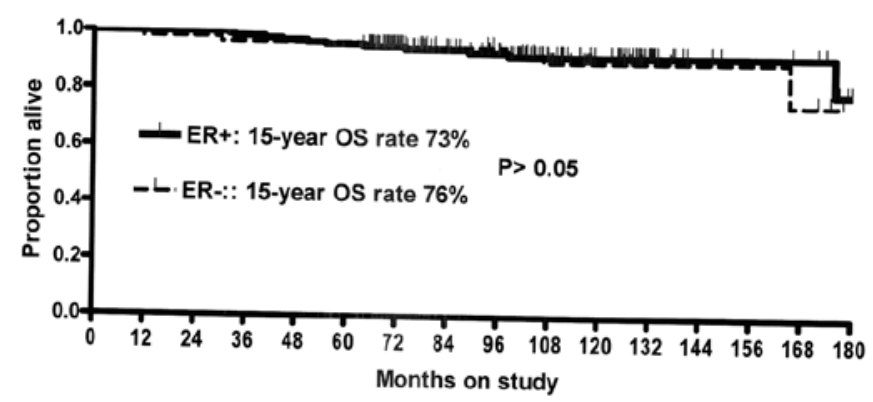

Figure 3. Overall survival (OS - ) of patients with estrogen receptor positive tumors $\left(\mathrm{ER}^{+}\right)$. OS (- $)$of patients with estrogen receptor negative $\left(\mathrm{ER}^{-}\right)$ tumors; $\mathrm{P}>0.05$ (Log-rank test).

recurrences, the median survival times were 50 months for those with $\mathrm{ER}^{+}$and 59 months for those with ER- tumors. The actuarial median time to progression and overall survival rates of all 200 patients (Fig. 1) have not been reached yet, as 64\% of patients were disease free and $73 \%$ were alive when the analysis of the data was performed. The projected DFS and OS rates at 10 years were 86 and $91 \%$, respectively. Patients with $\mathrm{ER}^{+}$tumors showed a statistically significant improvement in the 15-year DFS rate compared to patients with ER ${ }^{-}$tumors (Fig. 2). However, these groups showed no statistically significant difference in their 15-year OS rate (Fig. 3). Patients with positive and negative node tumors showed no statistically 
Table II. Grade 3 and 4 toxicity.

\begin{tabular}{|c|c|c|c|c|}
\hline & \multicolumn{4}{|c|}{ Type of therapy } \\
\hline & $\begin{array}{l}\text { LH-RH analogue } \\
\text { (200 patients) } \\
\text { No. }(\%)\end{array}$ & $\begin{array}{l}\text { Anthracycline-taxanes } \\
\text { (200 patients) } \\
\text { No. }(\%)\end{array}$ & $\begin{array}{c}\mathrm{CMF}+\mathrm{XRT} \\
(200 \text { patients }) \\
\text { No. }(\%)\end{array}$ & $\begin{array}{c}\text { HD-CT } \\
\text { (68 patients) } \\
\text { No. }(\%)\end{array}$ \\
\hline \multicolumn{5}{|l|}{ Haematologic } \\
\hline Leukopenia & 0 & $64(32 \%)$ & $6(3 \%)$ & $68(100 \%)$ \\
\hline Thrombocytopenia & 0 & $18 \quad(9 \%)$ & 0 & $68(100 \%)$ \\
\hline Anemia & 0 & $18 \quad(9 \%)$ & 0 & $4 \quad(6 \%)$ \\
\hline \multicolumn{5}{|l|}{ Gastrointestinal } \\
\hline Nausea-vomiting & 0 & $38(19 \%)$ & $26(13 \%)$ & $18 \quad(26 \%)$ \\
\hline Diarrhea & 0 & $12(6 \%)$ & $6(3 \%)$ & $9(13 \%)$ \\
\hline Mucositis & 0 & $10 \quad(5 \%)$ & $16(8 \%)$ & 0 \\
\hline Infection & 0 & $4 \quad(2 \%)$ & 0 & $3 \quad(4 \%)$ \\
\hline Neurotoxicity grade 2 & 0 & $20(10 \%)$ & 0 & 0 \\
\hline Alopecia & 0 & $200(100 \%)$ & 0 & $68(100 \%)$ \\
\hline Osteopenia & $40(20 \%)$ & & & \\
\hline Hot flushes & $180(90 \%)$ & 0 & 0 & 0 \\
\hline
\end{tabular}

CMF, cyclophosphamide, methotrexate, 5-fluorouracil. XRT, radiation therapy; HD-CT, dose-dense chemotherapy.
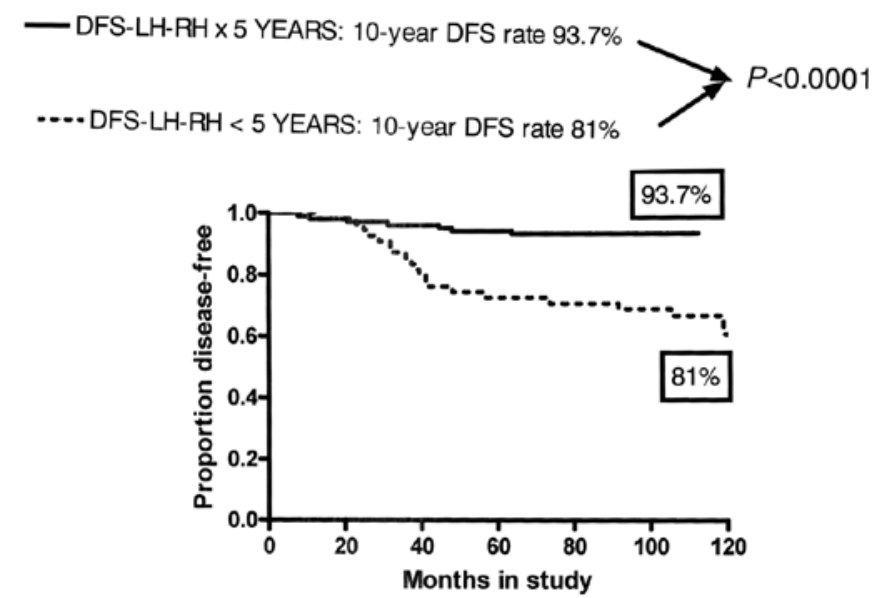

Figure 4. Ten-year disease-free survival rates. Patients were treated with the LH-RH analogue (goserelin) for 5 years $(\longrightarrow$ or $<5$ years $(--) ; \mathrm{P}<0.0001$.

significant difference in DFS and OS; however, patients with $>5$ positive axillary nodes had higher DFS $(\mathrm{P}<0.005)$ and OS $(\mathrm{P}<0.005)$ rates than patients with $<5$ involved nodes. Although there was no statistically significant difference in baseline characteristics, patients treated with the analogue for 5 years showed highly significant improvements in both DFS $(\mathrm{P}<0.0001)$ and OS $(\mathrm{P}<0.001)$ compared to patients treated for $<5$ years (Figs. 4 and 5 ).

Toxicity. Adverse events reported during the chemotherapeutic treatment are shown in Table II.

Goserelin. Fifty-six patients that received goserelin $(56 \%)$ complained of hot flushes.
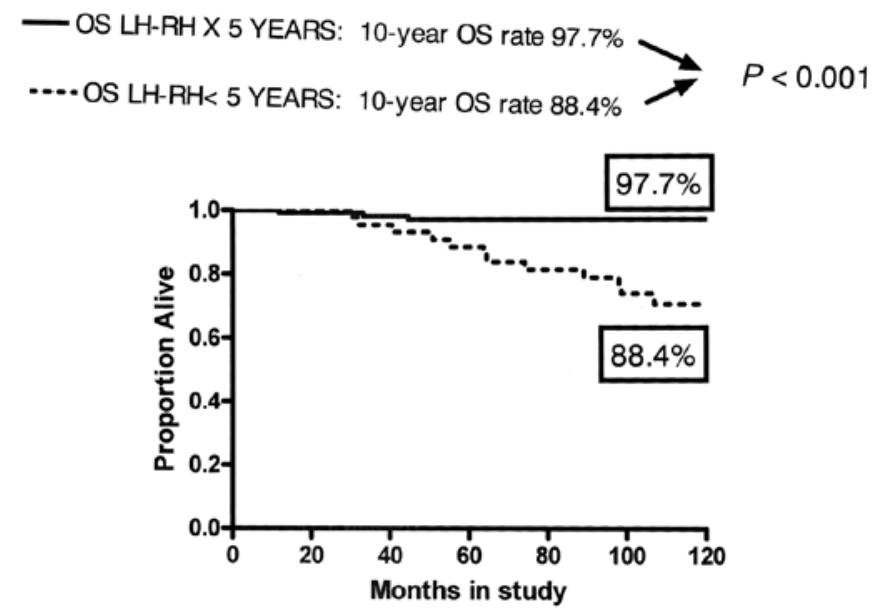

Figure 5. Ten-year overall survival rates. Patients were treated with the LH-RH analogue (goserelin) for 5 years $(-$ ) or $<5$ years $(---) ; \mathrm{P}<0.001$.

CMF chemotherapy. No unexpected toxicity occurred during the administration of CMF chemotherapy, and all 200 patients completed the scheduled treatment. Hematological toxicity grade 2 occurred in $23 \%$ of patients. Grade 2 diarrhea occurred in $10 \%$ of patients, and $31 \%$ of patients reported nausea and vomiting. There were no treatment-related deaths.

Anthracycline-taxane based chemotherapy. Grades 3-4 hematological toxicity occurred in $30 \%$ of patients treated with anthracycline-taxane based chemotherapy. Gastrointestinal toxicity symptoms were observed in 5\% (diarrhea) and 3\% (mucositis) of patients. Severe nausea and vomiting occurred in $13 \%$ of patients. Infection was reported in $3 \%$ of patients. Grade 3 alopecia was observed in all patients. No significant 
cardiac dysfunction was observed in any patient, and there were no treatment-related deaths.

High-dose chemotherapy. Nausea and vomiting and other gastrointestinal toxicity occurred in $30 \%$ of patients, but they only reached grades 2-3 severities, due to the appropriate use of antiemetic medications. Grade 4 neutropenia and thrombocytopenia were observed in all patients. An absolute neutrophil count of $<5 \times 10^{3} / \mathrm{ml}$ was observed for a median of 4.5 days (range, $3-5$ days), and a platelet count of $<20 \times 10^{3} / \mathrm{ml}$ occurred for a median of 1 day (range, 0-3 days). Six patients required a platelet transfusion (median of 2 units). Anemia, which occurred infrequently, due to the use of erythropoietin, occurred in $20 \%$ of patients. Six patients had fevers $>38^{\circ} \mathrm{C}$ for a median of 3 days (range, $0-6$ ). Grade 2 mucositis occurred in 6 patients, and grade 3 diarrhea occurred in 4 patients $(20 \%)$. One patient had a documented infection, where the blood culture was positive for staphylococcus epidermidis. Three patients reported bone pain for a median 2-day duration. There were no treatment-related deaths.

\section{Discussion}

Due to the high risk of recurrence in premenopausal breast cancer, we attempted to achieve the best results by combining all available treatment modalities, including surgery, hormonal therapy, chemotherapy and radiation therapy, as appropriate. After the surgical procedure, one week before starting adjuvant chemotherapy, an LH-RH analogue was administered to the second cohort of 100 patients. This therapy was continued for 5 years for a dual purpose: first, to protect the ovaries from the damage of chemotherapy, and second, to reduce the amount of circulating estrogen, a fundamental element for breast cancer cell survival (10). In fact, it has been shown that the hormonal surge associated with resumption or persistence of menses, after chemotherapy is a negative prognostic factor for DFS in premenopausal patients with early breast cancer (11). Radiation therapy was administered after chemotherapy, concurrent with the CMF regimen, to guarantee complete sterilization of the axilla and supraclavicular region $(12,13)$.

The use of chemotherapy in the adjuvant treatment of premenopausal breast cancer presents a trade-off between the benefit of reducing the odds of recurrence and the risk of damaging ovarian function and subsequent POF. Women have a fixed number of oocytes, which decreases with age (14). Hence, the severity of ovarian damage induced by chemotherapy depends on the age of the patient, the type of drug used, and the duration of exposure. Alkylating agents run the highest risk of ovarian damage, due to their highly reactive molecules. Agents that present intermediate risk include antibiotics, taxanes, anthracyclines and platinum derivates. Agents that are considered low risk include bleomycin, vinka alkaloids, and antimetabolites, such as 5-fluorouracil and methotrexate.

In recent years, progress in premenopausal breast cancer treatment has enabled some improvement in clinical outcome. In fact, in 1997, a randomized trial that enrolled 852 high-risk patients treated with combined CMF, chemotherapy, and radiation therapy, showed a 10-year OS rate of 54\% (15). In 2013, another trial with 3,312 patients that did not carry BRCA1, were treated with anthracycline-taxane-based chemotherapy and showed a 10 -year OS rate of $82.2 \%$ (16).

We used anthracycline-based adjuvant chemotherapy, because it has been shown to be more effective than CMF, and it offered the best results in premenopausal patients when administered in the sequence of adriamycin-CMF-goserelin (17). Additionally, the Early Breast Cancer Trialists' Collaborative Group has shown that 6 months of anthracycline-based polychemotherapy (e.g., with fluorouracil, cyclophosphamide and either doxorubicin or epirubicin) reduced the annual breast cancer-related death rate by $\sim 38 \%$ in women younger than 50 years of age. Those results were largely independent of tamoxifen use, estrogen receptor status, nodal status, or other tumor characteristics. Furthermore, for $\mathrm{ER}^{+}$disease only, 5 years of adjuvant tamoxifen reduced the annual breast cancerrelated death rate by $31 \%$ (7).

Several studies have addressed the role of LH-RH analogues during adjuvant chemotherapy. Adjuvant chemotherapy with CMF was compared directly with goserelin in the treatment of premenopausal, node-negative breast cancer. Patients with ER- tumors achieved higher DFS rates when they received CMF than when they received goserelin alone (84 vs. $73 \%$ ). In contrast, in patients with $\mathrm{ER}^{+}$disease, chemotherapy alone and goserelin alone produced similar results (18). Complete hormonal blockade with an LH-RH analogue and tamoxifen for three years was compared to the fluorouracil, epirubicin, and cyclophosphamide regimen in a group of 333 patients with intermediate risk breast cancer. That randomized study found no statistically significant difference in either DFS or OS between the two groups of patients (19). In another study, goserelin alone did not significantly reduce recurrence or death after recurrence in patients with hormone-receptorpositive cancers; however, the addition of LH-RH agonists to tamoxifen, chemotherapy, or both reduced recurrence by $12.7 \%$ and death after recurrence by $15.1 \%$. In that group of patients, the addition of LH-RH agonists showed similar efficacy to chemotherapy alone. However, the duration of LH-RH analogue administration was not assessed. Moreover, no trial had assessed an LH-RH agonist vs. chemotherapy. However, LH-RH agonists were shown to be ineffective in hormonereceptor-negative tumors (20).

Previous studies have reported conflicting results regarding the utility of LH-RH analogues for avoiding POF. A recently published study found that it was uncertain whether LH-RH agonists were useful for preserving fertility in young patients with breast cancer (21). A recent meta-analysis calculated a pooled risk estimate and found a highly significant reduction in the risk of POF (OR, 0.43; 95\% CI, 0.22-0.84; $\mathrm{P}=0.013$ ) in patients that received LH-RH analogues (22).

More recently, attention has focused on the potential effects of LH-RH analogues on survival of premenopausal patients with breast cancer. Two recent studies have shed light on the utility of LH-RH analogues in treating premenopausal patients with breast cancers that were $\mathrm{ER}^{+}(23)$ and $\mathrm{ER}^{-}(24)$. In the first study (2003-2011), 4,690 premenopausal women with hormone-receptor-positive early breast cancer were randomized to receive either exemestane plus ovarian suppression or tamoxifen plus ovarian suppression. After a median follow-up of 68 months, patients that received exemestane plus ovarian suppression showed significant reductions in recurrence 
compared to patients that received tamoxifen plus ovarian suppression. Moreover, the 5-year DFS rates were 91.1 and $87.3 \%$, respectively $(\mathrm{P}<0.001)$, and $\mathrm{OS}$ rates were 95.9 and $96.9 \%$, respectively $(\mathrm{P}=0.37)$. However, it should be noted that $57.8 \%$ of patients in that study (23) had negative axillary nodes. In addition, a large proportion of patients in that study (1,996 patients) had low risk of recurrence and did not receive chemotherapy.

The second study was the phase III Prevention of Early Menopause Study (POEMS)/Southwest Oncology Group (SWOG) S0230 (2004 to 2011). That study evaluated the rate of POF in premenopausal women, aged 18-49, with stage I to IIIA ER ${ }^{-}$and PR $^{-}$breast cancers (24). After considering all the drop outs, the remaining fully evaluable patients included 69 randomized to chemotherapy alone and 66 randomized to chemotherapy plus goserelin. At 2 years, $21 \%$ of patients treated with chemotherapy had POF, and only $7.5 \%$ of patients treated with chemotherapy plus goserelin had POF, which represented a $70 \%$ reduction. Patients treated with chemotherapy alone had 4-year DFS and OS rates of 78 and 82\%, respectively; patients treated with chemotherapy plus goserelin had 4-year DFS and OS rates of 89 and $92 \%$, respectively.

Although the patients in the present study were not randomized, they reflected a population of patients at midhigh risk for breast cancer that were recruited sequentially in a general community hospital. We analyzed the potential benefit of LH-RH analogues in preventing POF with a long follow-up; the median was 105 months (range, 65-180). For comparison, the two studies described above had median follow-ups of 68 months (23) and 49.2 months (24). Our 4-year DFS and OS rates in patients with $\mathrm{ER}^{-}$tumors were 84 and $96 \%$, respectively. Our 5-year DFS and OS rates in patients with $\mathrm{ER}^{+}$tumors were 92 and $96 \%$, respectively. The entire cohort had 10-year DFS and OS rates of 85 and 91\%, respectively, and 15-year DFS and OS rates of 64 and 73\%, respectively. Among our cohort of 200 patients, 142 patients with $\mathrm{N}^{+}$and $\mathrm{ER}^{+}$tumors had 9-year DFS and OS rates of 91 and $92 \%$, respectively. These rates were consistent with a cohort of 507 patients, described in 2005, that had 9-year DFS and OS rates of 68 and $76 \%$, respectively (25).

In conclusion, the present phase II trial had a much longer follow-up than previous studies of this kind. We confirmed the results obtained in a previous randomized trial (23) for premenopausal patients with hormone receptor positive tumors, which showed that the addition of an LH-RH analogue to adjuvant chemotherapy, followed by examestane, provided a new treatment option that reduced the risk of recurrence. We also confirmed previous findings that the same treatment reduced the risk of breast cancer recurrence in premenopausal patients with hormone receptor negative tumors (24).

\section{References}

1. Beatson GT: On the treatment of inoperable cases of carcinoma of the mamma: suggestions for a new method of treatment with illustrative cases. Lancet ii: 104-107, 1896.

2. Clarke MG: Ovarian ablation in breast cancer 1896-1998. Milestones along hierarchy from evidence from case report to Chochrane review. BMJ 317: 1246-1248, 1998

3. Adjuvant chemotherapy for breast cancer. NIH Consensus Statement Online 5: 1-19, 1985.
4. Bines J, Oleske DM and Cobleigh MA: Ovarian function in premenopausal women treated with adjuvant chemotherapy for breast cancer. J Clin Oncol 14: 1718-1729, 1996.

5. Recchia F, Sica G, De Filippis S, Rosselli M and Rea S: Goserelin as ovarian proitection in the adjuvant treatment of premenopausal breast cancer: a phase II pilot study. Anticancer Drugs 13: 417-424, 2001.

6. Recchia F, Saggio G, Amiconi G, Di Blasio A, Cesta A, Candeloro $\mathrm{G}$ and Rea S: Gonadotropin-releasing hormone analogues added to adjuvant chemotherapy protect ovarian function and improve clinical outcomes in young women with early breast carcinoma. Cancer 106: 514-523, 2006.

7. Early Breast Cancer Trialists' Collaborative Group (EBCTCG): Effects of chemotherapy and hormonal therapy for early breast cancer on recurrence and 15-year survival: an overview of the randomised trials. Lancet 365: 1687-1717, 2005.

8. Kaplan EL and Meier P: Nonparametric estimation from incomplete observations. J Am Stat Ass 53: 457-481, 1958.

9. Therasse P, Arbuck SG, Eisenhauer EA, et al: New guidelines to evaluate the response to treatment in solid tumors: European Organization for Research and Treatment of Cancer, National Cancer Institute of the United States, National Cancer Institute of Canada. J Natl Cancer Inst 92: 205-216, 2000.

10. Endogenous Hormones and Breast Cancer Collaborative Group; Key TJ, Appleby PN, Reeves GK, et al: Sex hormones and risk of breast cancer in premenopausal women: a collaborative reanalysis of individual participant data from seven prospective studies. Lancet Oncol 14: 1009-1019, 2013.

11. Park IH, Han HS, Lee H, et al: Resumption or persistence of menstruation after cytotoxic chemotherapy is a prognostic factor for poor disease-free survival in premenopausal patients with early breast cancer. Ann Oncol 23: 2283-2289, 2012.

12. Ragaz J, Jackson SM, Le N, et al: Adjuvant radiotherapy and chemotherapy in node-positive premenopausal women with breast cancer. N Engl J Med 337: 956-962, 1997.

13. Toledano A, Azria D, Garaud P, et al: Phase III trial of concurrent or sequential adjuvant chemoradiotherapy after conservative surgery for early-stage breast cancer: final results of the ARCOSEIN trial. J Clin Oncol 25: 405-410, 2007.

14. Meirow D, Biederman H, Anderson RA and Wallace WH: Toxicity of chemotherapy and radiation on female reproduction. Clin Obstet Gynecol 53: 727-739, 2010.

15. Overgaard M, Hansen PS, Overgaard J, et al: for the Danish Breast Cancer Cooperative Group 82b Trial: Postoperative radiotherapy in high-risk premenopausal women with breast cancer who receive adjuvant chemotherapy. New Eng J Med 337: 949-955, 1997.

16. Huzarski T, Byrski T, Gronwald J, et al: Ten-year survival in patients with BRCA1-negative and BRCA1-positive breast cancer. J Clin Oncol 31: 3191-3196, 2013.

17. De Placido S, De Laurentiis M, De Lena M, et al: GOCSI Cooperative Group: A randomised factorial trial of sequential doxorubicin and CMF vs CMF and chemotherapy alone vs chemotherapy followed by goserelin plus tamoxifen as adjuvant treatment of node-positive breast cancer. Br J Cancer 92: 467-474, 2005.

18. International Breast Cancer Study Group (IBCSG); CastiglioneGertsch M, O'Neill A, Price KN, et al: Adjuvant chemotherapy followed by goserelin versus either modality alone for premenopausal lymph node-negative breast cancer: a randomized trial. J Natl Cancer Inst 95: 1833-1846, 2003.

19. Roché H, Kerbrat P, Bonneterre J, et al: Complete hormonal blockade versus epirubicin-based chemotherapy in premenopausal, one to three node-positive, and hormone-receptor positive, early breast cancer patients: 7-year follow-up results of French Adjuvant Study Group 06 randomised trial. Ann Oncol 17: 1221-1227, 2006.

20. LHRH-agonists in Early Breast Cancer Overview Group; Cuzick J, Ambroisine L, Davidson N, Jakesz R, Kaufmann M, Regan M and Sainsbury R: Use of luteinising-hormone-releasing hormone agonists as adjuvant treatment in premenopausal patients with hormone-receptor-positive breast cancer: a metaanalysis of individual patient data from randomised adjuvant trials. Lancet 369: 1711-1723, 2007.

21. Turner NH, Partridge A, Sanna G, Di Leo A and Biganzoli L: Utility of gonadotropin-releasing hormone agonists for fertility preservation in young breast cancer patients:the benefit remains uncertain. Ann Oncol 24: 2224-2235, 2013. 
22. Del Mastro L, Ceppi M, Poggio F, et al: Gonadotropin-releasing hormone analogues for the prevention of chemotherapy-induced premature ovarian failure in cancer women: systematic review and meta-analysis of randomized trials. Cancer Treat Rev 40: 675-683, 2014

23. Pagani O, Regan MM, Walley BA, et al; TEXT and SOFT Investigators and the International Breast Cancer Study Group: Adjuvant exemestane with ovarian suppression in premenopausal breast cancer. N Engl J Med 371: 107-118, 2014.
24. Moore HCF, Unger JM, Phillips KA, et al: Phase III trial of LHRH analog during chemotherapy to reduce ovarian failure in early-stage, hormone receptor-negative breast cancer. ASCO Annual Meeting. Presented May 31, 2014. J Clin Oncol 32 (5s): LBA505, 2014.

25. Davidson NE, O'Neill AM, Vukov AM, Osborne CK, Martino S, White DR and Abeloff MD: Chemoendocrine therapy for premenopausal women with axillary lymph node positive, steroid hormone receptor-positive breast cancer: results from INT 0101 (E5188). J Clin Oncol 23: 5973-5982, 2005. 